\title{
Persistent spin current in anisotropic spin ring
}

\author{
Yin Cheng and You-Quan Li \\ Zhejiang Institute of Modern Physics, \\ Zhejiang University, Hangzhou 310027, P.R. China \\ Bin Chen \\ Department of Physics, Hangzhou Teacher's College, Hangzhou 310036, P.R. China
}

(Received December 14, 2018)

\begin{abstract}
The persistent spin current in anisotropic spin ring penetrated by a $\mathrm{SU}(2)$ flux is studied by the Schwinger-boson mean field approach. The anisotropy in spin coupling can facilitate the persistent spin current. Ground-state energy and excitation energy gap are also studied. The peak of spin current occurs at the maximum value of the ground-state energy.
\end{abstract}




\section{INTRODUCTION}

The quantum coherence plays a central role in mesoscopic physics and the persistent current on mesoscopic rings threaded by a magnetic flux is a particular sensitive probe of such coherence. Thus there has been much study on persistent electrical current in mesoscopic ring both experimentally [1, 2, 3] and theoretically [4, 5, 6, 6]. Owning to recent interests in the spin based electronics [8], the study on spin current becomes a remarkable topic [9, 10, 11, 12, 13]. The persistent spin current in the ferromagnetic Heisenberg ring was shown to occur in the presence of crown-shaped magnetic field [14]. It can also be driven by inhomogeneous electric fields [15] due to the Aharonov-Casher effect [16]. On the basis of spin-wave approach, spin current in antiferromagnetic Heisenberg ring in inhomogeneous magnetic field has been investigated very recently [17]. It is well-known that the electrical persistent current is an topological current produced by the magnetic flux, a "U(1) flux". However, as far as we were aware, there is not a thorough discussion about persistent spin current in the anisotropic Heisenberg model produced by a $\mathrm{SU}(2)$ flux.

In this paper, we study the anisotropy Heisenberg rings (XXZ model) penetrated by a SU(2) flux. In Sec we apply the Schwinger-boson approach to the model. In Sec III we calculate the excitation spectrum and obtain the ground-state energy and energy gap. In SecIV, we evaluate the persistent spin current and discuss the effects caused by the

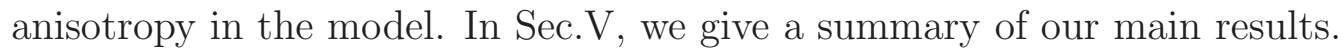

\section{SCHWINGER-BOSON APPROACH}

We consider a spin ring with anisotropy penetrated by the $\mathrm{SU}(2)$ flux:

$$
H=\sum_{j}^{N}\left[\frac{1}{2}\left(e^{i \phi / N} s_{j}^{+} s_{j+1}^{-}+e^{-i \phi / N} s_{j}^{-} s_{j+1}^{+}\right) \Delta s_{j}^{z} s_{j+1}^{z}\right]+h . c .
$$

where $s_{j}^{ \pm}$denote for spin flipping operarors and $s_{j}^{z}$ for the $z$ component of spin operator; $\phi=\Phi / \Phi_{0}$ with $\Phi$ and $\Phi_{0}(=h c / e)$ the $\mathrm{SU}(2)$ flux and the flux quanta respectively; $N$ the lattice number. The anisotropy is characterized by the parameter $\Delta$. As is known that $\Delta=-1$ corresponds to the ferromagnetic case, $\Delta=1$ is the anti-ferromagnetic regime and $-1<\Delta<1$ is the transition regime from ferromagnetic to anti-ferromagnetic.

In terms of Schwinger boson operators $a_{j}$ and $b_{j}$ which satisfy the Bose commutation 
relations $\left[a_{i}, a_{j}^{\dagger}\right]=\delta_{i j}$ and $\left[a_{i}, b_{j}\right]=0$, the spin operators are given by

$$
s_{j}^{+}=a_{j}^{\dagger} b_{j}, \quad s_{j}^{-}=a_{j} b_{j}^{\dagger}, \quad s_{j}^{z}=\frac{1}{2}\left(a_{j}^{\dagger} a_{j}-b_{j}^{\dagger} b_{j}\right)
$$

with a local constraint at every site $j$ given by $a_{j}^{\dagger} a_{j}+b_{j}^{\dagger} b_{j}=2 S$ which means only $2 S$ of the two bosons can occupy each site.

Since the lattice is a bipartite lattice, we can make a unitary transformation $a_{j+1} \rightarrow$ $-a_{j+1}, b_{j+1} \rightarrow b_{j+1}$ at each site of one sublattice. This brings about $s_{j+1}^{ \pm} \rightarrow-s_{j+1}^{ \pm}$and $s_{j+1}^{z} \rightarrow s_{j+1}^{z}$. The XXZ Hamiltonian (1) becomes the following form:

$$
\begin{aligned}
H & =\frac{1}{2} \sum_{j}\left[\left(-e^{i \phi / N} a_{j}^{\dagger} b_{j} a_{j+1} b_{j+1}^{\dagger}-e^{-i \phi / N} a_{j} b_{j}^{\dagger} a_{j+1}^{\dagger} b_{j+1}\right),\right. \\
& \left.+\frac{\Delta}{2}\left(a_{j}^{\dagger} a_{j} a_{j+1}^{\dagger} a_{j+1}-a_{j}^{\dagger} a_{j} b_{j+1}^{\dagger} b_{j+1}-b_{j}^{\dagger} b_{j} a_{j+1}^{\dagger} a_{j+1}+b_{j}^{\dagger} b_{j} b_{j+1}^{\dagger} b_{j+1}\right)\right]+ \text { h.c. }
\end{aligned}
$$

To fulfil the constraint, we need to introduce a Lagrangian-multiplier field $\lambda_{i}$. Then a generalized Hamiltonian is obtained

$$
\begin{aligned}
H=-\sum_{j} & \left\{\left[\frac{1-\Delta}{4} \mathcal{A}_{j, j+1}^{\dagger} \mathcal{A}_{j, j+1}+\frac{1+\Delta}{4} \mathcal{B}_{j, j+1}^{\dagger} \mathcal{B}_{j, j+1}\right]+h . c .\right\} \\
+ & 2 \sum_{j} \lambda_{i}\left(a_{j}^{\dagger} a_{j}+b_{j}^{\dagger} b_{j}-2 S\right)+2 N S^{2}+(1-\Delta) N S .
\end{aligned}
$$

where

$$
\begin{aligned}
& \mathcal{A}_{j, j+1}^{\dagger}=e^{-i \phi / 2 N} a_{j} a_{j+1}^{\dagger}+e^{i \phi / 2 N} b_{j} b_{j+1}^{\dagger}, \\
& \mathcal{B}_{j, j+1}^{\dagger}=e^{i \phi / 2 N} a_{j}^{\dagger} b_{j+1}^{\dagger}+e^{-i \phi / 2 N} b_{j}^{\dagger} a_{j+1}^{\dagger} .
\end{aligned}
$$

The isotropy limits $\Delta= \pm 1$ (i.e., ferro- and antiferromagnetic cases) without flux were considered in Ref. [18]. At the mean-field level, we take the average value of the multiplier field $\left\langle\lambda_{i}\right\rangle=\lambda$ and make the bond operators $\left\langle\frac{1-\Delta}{4} \mathcal{A}_{j, j+1}^{\dagger}\right\rangle=A^{*}$ and $\left\langle\frac{1+\Delta}{4} \mathcal{B}_{j, j+1}^{\dagger}\right\rangle=B^{*}$ uniform and static. We hence obtain the mean-field Hamiltonian:

$$
\begin{aligned}
H_{\mathrm{MF}} & =-\sum_{j}\left\{\left[\left(A^{*} \cdot \mathcal{A}_{j, j+1}+A \cdot \mathcal{A}_{j, j+1}^{\dagger}\right)+\left(B^{*} \cdot \mathcal{B}_{j, j+1}+B \cdot \mathcal{B}_{j, j+1}^{\dagger}\right)\right]+\text { h.c. }\right\}, \\
& +2 \lambda \sum_{j}\left(a_{j}^{\dagger} a_{j}+b_{j}^{\dagger} b_{j}-2 S\right)+\frac{8}{1-\Delta} A^{*} A N+\frac{8}{1+\Delta} B^{*} B+2 N S^{2}+(1-\Delta) N S .(5)
\end{aligned}
$$


By making use of a Fourier transform $a_{j}=\Sigma_{\mathbf{k}} a_{\mathbf{k}} \exp \left(i \mathbf{k} r_{j}\right)$ where the summation $\mathbf{k}$ runs over the first Brillouin zone, a four-component spinor

$$
\Psi_{\mathbf{k}}^{\dagger}=\left(a_{\mathbf{k}}^{\dagger}, a_{\mathbf{k}}, b_{-\mathbf{k}}^{\dagger}, b_{-\mathbf{k}}\right)
$$

can be introduced. We can write the mean-field Hamiltonian into a compact form in the momentum space:

$$
H_{\mathrm{MF}}=\sum_{\mathbf{k}}\left\{\Psi_{\mathbf{k}}^{\dagger}\left[\lambda-2 \cos \left(\mathbf{k}+\frac{\phi}{2 N}\right) \mathcal{M}\right] \Psi_{\mathbf{k}}+2 A \cos \left(\mathbf{k}+\frac{\phi}{2 N}\right)\right\}+\varepsilon_{0},
$$

where

$$
\varepsilon_{0}=\frac{8}{1-\Delta} A^{*} A N+\frac{8}{1+\Delta} B^{*} B N+(1-\Delta) N S+2 N S^{2}-2 \lambda N(2 S+1),
$$

and

$$
\mathcal{M}=\left(\begin{array}{cccc}
A^{*} & 0 & 0 & B \\
0 & A & B^{*} & 0 \\
0 & B & A^{*} & 0 \\
B^{*} & 0 & 0 & A
\end{array}\right)
$$

The Hermitian property of the Hamiltonian (17) enables us easily to obtain that $A=A^{*}$. Using a Bogoliubov transformation given by the following transformation matrix $\mathcal{T}$ :

$$
\mathcal{T}=\left(\begin{array}{llll}
u & 0 & 0 & v \\
0 & u & v & 0 \\
0 & v & u & 0 \\
v & 0 & 0 & u
\end{array}\right),
$$

we transform the original Bose operators $\left\{a_{\mathbf{k}}^{\dagger}, a_{\mathbf{k}}, b_{-\mathbf{k}}^{\dagger}, b_{-\mathbf{k}}\right\}$ to a set of new Bose operators, called "quasi-particle" creation/anihilation operators, $\left\{\alpha_{\mathbf{k}}^{\dagger}, \alpha_{\mathbf{k}}, \beta_{\mathbf{k}}^{\dagger}, \beta_{\mathbf{k}}\right\}$

$$
\mathcal{T}\left(\begin{array}{c}
a_{\mathbf{k}}^{\dagger} \\
a_{\mathbf{k}} \\
b_{-\mathbf{k}}^{\dagger} \\
b_{-\mathbf{k}}
\end{array}\right)=\left(\begin{array}{c}
\alpha_{\mathbf{k}}^{\dagger} \\
\alpha_{\mathbf{k}} \\
\beta_{\mathbf{k}}^{\dagger} \\
\beta_{\mathbf{k}}
\end{array}\right) .
$$


Then the Hamiltonian is diagonalized,

$$
H_{\mathrm{MF}}=\sum_{\mathbf{k}}\left[\omega_{\mathbf{k}, \phi}\left(\alpha_{\mathbf{k}}^{\dagger} \alpha_{\mathbf{k}}+\beta_{k}^{\dagger} \beta_{\mathbf{k}}+1\right)+2 A \cos \left(\mathbf{k}+\frac{\phi}{2 N}\right)\right]+\varepsilon_{0},
$$

where the quasi-particle spectrum is

$$
\omega_{\mathbf{k}, \phi}=\sqrt{\left[\lambda-2 A \cos \left(\mathbf{k}+\frac{\phi}{2 N}\right)\right]^{2}-\left|2 B \cos \left(\mathbf{k}+\frac{\phi}{2 N}\right)\right|^{2}} .
$$

Thus the free energy is obtained

$$
f=\frac{F_{\mathrm{MF}}}{2 N}=\int_{-\frac{\pi}{2}}^{\frac{\pi}{2}} \frac{\mathrm{d} \mathbf{k}}{2 \pi}\left[\frac{2}{\beta} \ln \left(2 \sinh \frac{\beta \omega_{\mathbf{k}, \phi}}{2}\right)+2 A \cos \left(\mathbf{k}+\frac{\phi}{2 N}\right)\right]+\frac{\varepsilon_{0}}{2 N} .
$$

where $\beta=1 / k_{B} T$ with $k_{B}$ the Boltzmann constant and $T$ the temperature. The mean-

field self-consistent equations are obtained by minimizing the free energy, i.e., $\delta f / \delta A=$ $0, \delta f / \delta B=0, \delta f / \delta \lambda=0$, then the saddle-point equations are given by

$$
\begin{aligned}
& A+\frac{1-\Delta}{4} \int_{-\frac{\pi}{2}}^{\frac{\pi}{2}} \frac{\mathrm{d} \mathbf{k}}{2 \pi}\left[1-\frac{\lambda-2 A \cos \left(\mathbf{k}+\frac{\phi}{2 N}\right)}{\omega_{\mathbf{k}, \phi}} \cdot \operatorname{coth}\left(\frac{\beta \omega_{\mathbf{k}, \phi}}{2}\right)\right] \cdot \cos \left(\mathbf{k}+\frac{\phi}{2 N}\right)=0 \\
& 1-\frac{1+\Delta}{2} \int_{-\frac{\pi}{2}}^{\frac{\pi}{2}} \frac{\mathrm{d} \mathbf{k}}{2 \pi}\left[\frac{\left|\cos \left(\mathbf{k}+\frac{\phi}{2 N}\right)\right|^{2}}{\omega_{\mathbf{k}, \phi}} \cdot \operatorname{coth}\left(\frac{\beta \omega_{\mathbf{k}, \phi}}{2}\right)\right]=0 \\
& (2 S+1)-\int_{-\frac{\pi}{2}}^{\frac{\pi}{2}} \frac{\mathrm{d} \mathbf{k}}{2 \pi}\left[\frac{\lambda-2 A \cos \left(\mathbf{k}+\frac{\phi}{2 N}\right)}{\omega_{\mathbf{k}, \phi}} \cdot \operatorname{coth}\left(\frac{\beta \omega_{\mathbf{k}, \phi}}{2}\right)\right]=0
\end{aligned}
$$

which determine the parameters $A, B$ and the Lagrange multiplier (chemical potential) $\lambda$ in Eqs. (11)-(12). These saddle point equations can be solved numerically.

\section{THE GROUND-STATE ENERGY AND ENERGY GAP}

We consider $S=1$ at $T=0 \mathrm{~K}$ with $N=50$ sites. At zero temperature, the groundstate (GS) energy equals to the free energy because there is no thermal fluctuation. When $T \rightarrow 0^{+}, \beta$ tends to $+\infty$ which means $e^{-\beta \omega_{\mathbf{k}, \phi} / 2} \rightarrow 0^{+}$if $\omega_{\mathbf{k}, \phi}$ is finite. Thus in the first term of the integral in the free energy equation (12) $\ln \left(2 \sinh \frac{\beta}{2} \omega_{\mathbf{k}, \phi}\right)$ reduces to $\omega_{\mathbf{k}, \phi}$ and the free energy (1.e., ground-state energy per site at $T=0$ ) becomes

$$
E_{\mathrm{GS}}(\phi)=\int_{-\frac{\pi}{2}}^{\frac{\pi}{2}} \frac{\mathrm{d} \mathbf{k}}{2 \pi}\left[\omega_{\mathbf{k}, \phi}+2 A \cos \left(\mathbf{k}+\frac{\phi}{2 N}\right)\right]+\frac{\varepsilon_{o}}{N}
$$


We plot the ground-state energy per site versus $\theta$ (here we set $\phi / N=\theta$ ) for $\Delta=0.9$ in Fig. 1. Apparently, the ground-state energy has a sharp cusp at $\theta / 2 \pi= \pm 0.5$ whose magnitude is -0.9626 , which is higher than average value of the energy gap. The height of cusp peak

raises while $\Delta$ decreases, which is shown in the inset of the Fig. 1 In other words, more anisotropy of spin chain results in sharper cusp in the curve.

We calculate the energy gap (EG) of the first excited state over the ground state. Unlike the usual case, the energy gap is not at $\mathbf{k}=0$ due to the presence of the external $\mathrm{SU}(2)$ flux. This implies that the magnitude of the gap changes with the flux accordingly, which is shown in Fig. 2. Clearly, $\mathbf{k}$ undergoes a jump at $\theta / 2 \pi= \pm 0.5$. The Fig. 3] shows the excitation energy gap versus the magnetic flux. The gap descents as the flux goes to $\theta / 2 \pi= \pm 0.5$, but rebounds when it is very close to $\theta / 2 \pi= \pm 0.5$ and reaches the maximum at $\theta / 2 \pi= \pm 0.5$.

\section{PERSISTENT SPIN CURRENT}

We have previously obtained the ground-state energy and excitation spectrum whose values are determined by Eqs.(13). Now we are in the position to evaluate the persistent current at zero temperature $(T=0 \mathrm{~K})$ which is defined by

$$
I(\phi, T)=-\frac{\partial E_{\mathrm{GS}}(\phi)}{\partial \phi},
$$

where $E_{\mathrm{GS}}(\phi)$ is the ground-state energy Eq. (14). The charge current $I_{c}$ is null in our model for the particle is fixed on each site. However, the pure persistent spin current in the ring can be deduced by the SU(2) flux. We plot numerical calculation of Eq. (15) in Fig. 4 (a). The jumps in the spin current curve occur at $\theta / 2 \pi= \pm 0.5$ for various $\Delta$. We found that the anisotropy $(\Delta)$ will enhance the persistent spin current, which is shown in (b) of Fig 4. As a result, the spin current in $X Y$ limit is larger than in Heisenberg limit. Fig. 5 exhibits that the energy gap decreases while persistent current increases. The larger the energy gap is, the more spin flippings that contributes to spin current are prevented.

\section{SUMMARY AND DISCUSSION}

Using Schwinger-bonson mean field approach, we have investigated the property of ground-state and energy gap for the anisotropy spin ring penetrated by $\mathrm{SU}(2)$ flux. In 
the curve of energy versus flux, there is a cusp at $\theta / 2 \pi= \pm 0.5$ and the energy reaches a maximum. Whereas, the excitation energy gap drops drastically nearby $\theta / 2 \pi= \pm 0.5$ but rebound to maxima at $\theta / 2 \pi= \pm 0.5$. This implies that the energy curve of the first excited state has a similar shape as Fig. 1 and is tangent to the curve of the ground state near the point $\theta= \pm 0.5$. We calculated the pure persistent spin current and found that the flux dependence of persistent spin currents are facilitated by the anisotropy parameter $\Delta$ which promotes the persistent spin current. The peak of spin current appears at the minimal value of excitation gap.

The work is supported by NSFC grant No.10225419.

[1] L. P. Levy, G. Dolan, J. Dunsmuir and H. Bouchiat, Phys. Rev. Lett. 64 (1990) 2074.

[2] V. Chandrasekhar, R. A. Webb, M. J. Brady, M. B. Ketchen, W. J. Gallagher and A. Kleinsasser, Phys. Rev. Lett. 67, 3578 (1991).

[3] D. Mailly, C. Chapelier and A. Benoit, Phys. Rev. Lett. 70, 2020 (1993).

[4] M. Büttiker, Y. Imry and R. Landauer, Phys. Lett. A96, 365 (1983).

[5] V. Ambegaokar and U. Echern, Phys. Rev. Lett. 65, 381 (1990). U. Eckern and A. Schmid, Europhys. Lett. 18, 457 (1992).

[6] Y. Q. Li and Z. S. Ma, J. Phys. Soc. Jpn. 65, 1519 (1996).

[7] P. Koskinen, and M. Manninen, phys. rev. B 68, 195304 (2003).

[8] Awschalom, Loss, Samarth, Semiconductor spintronics and quantum computation, ( Springer 2002, New York)

[9] S. Murakami, N. Nagaosa, and S. C. Zhang, Science 301 1348-1351 (2003); ibid. Phys. Rev. B 69, 235206 (2004).

[10] J. Sinova, D. Culcer, Q. Niu, N. A. Sinitsyn, T. Jungwirth, and A. H. MacDonald, Phys. Rev. Lett. 92, 126603 (2004).

[11] E. G. Mishchenko, A. V. Shytov, and B. I. Halperin, Phys. Rev. Lett. 93, 226602 (2004).

[12] P. Q. Jin, Y. Q. Li, and F. C. Zhang, cond-mat/0502231

[13] J. Splettstoesser, M. Goveruals, and U. Zülicke, phsy. Rev. B 68, 165341 (2003).

[14] F. Schütz, M. Kollar, and P. Kopietz, Phys. Rev. Lett. 91017205 (2003).

[15] Z.L. Cao, X.P. Yu, and R.S. Han, Phys. Rev. B 56, 5077 (1997). 
[16] Y. Aharonov, and A. Casher, Phys. Rev. Lett. 53, 319(1984).

[17] F. Schütz, M. Kollar, and P. Kopietz, Phys. Rev. B 69, 035313 (2004).

[18] Charles J. De Leone, and Gergely T. Zimanyi Phys. Rev. B 49, 1131 (1994). 


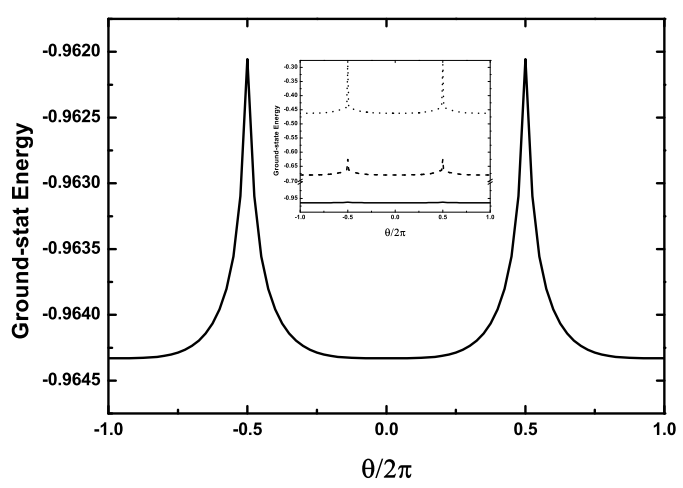

FIG. 1: The ground-state energy versus $\mathrm{SU}(2)$ flux $\theta(\theta=\phi / N)$ for $\Delta=0.9$. The insert shows ground-state energy for various anisotropy $\Delta=0.1$ (dot), $\Delta=0.5$ (dash) and $\Delta=0.9$ (solid).



FIG. 2: Momentum versus flux for $\Delta=0.9$. 


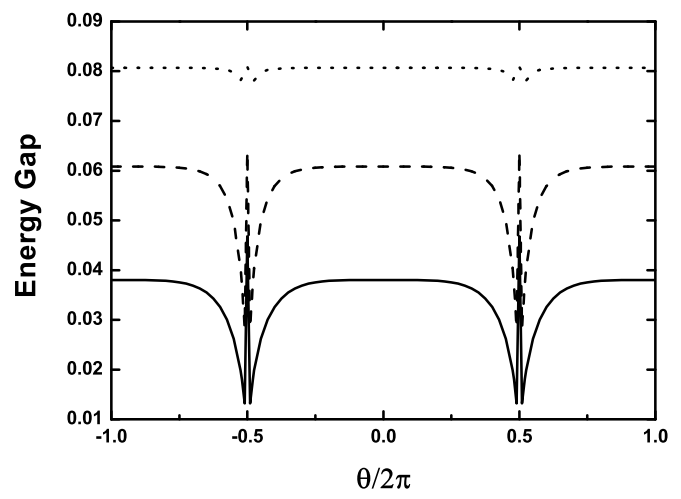

FIG. 3: The excitation energy gap versus flux for $\Delta=0.1$ (solid), $\Delta=0.5$ (dash) and $\Delta=0.9$ (dot).


FIG. 4: (a) Persistent spin current versus flux for various anisotropy parameter $\Delta$. The dash-, solid-, and dot-line corresponds to $\Delta=0.1,0.5$ and 0.9 respectively. (b) The current versus $\Delta$ illustrates the effects caused by anisotropy parameter. 


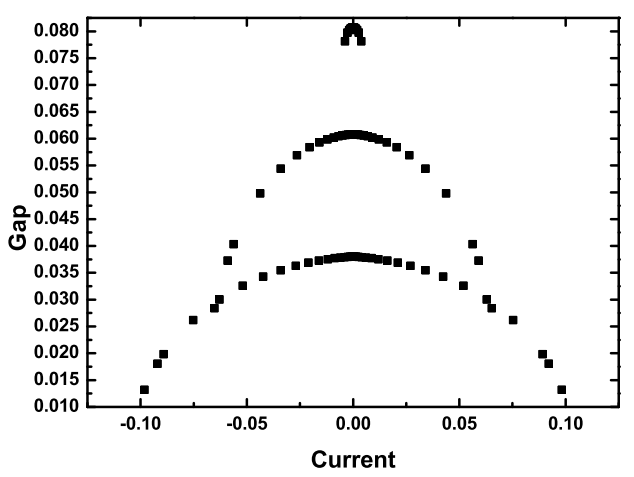

FIG. 5: Energy gap versus persistent spin current for different anisotropy parameters $\Delta$. Hollow $\operatorname{circle}(\circ), \operatorname{cross}(\times)$ and solid $\operatorname{circle}(\bullet)$ corresponds to $\Delta=0.1,0.5$ and 0.9 respectively. 